\title{
Design of a Resilience Model Based on the Institutionalization of Organizational Learning in the Red Crescent Society
}

\author{
Alireza Ebrahimi ${ }^{1}$ (1) \\ Date of submission: 8 Nov. 2019 Date of acceptance: 17 Mar. 2020
}

\begin{abstract}
INTRODUCTION: In Iran, natural disasters are constantly increasing; however, no research has clearly explained the indicators of resilience in such situations. Resilience enhances internal strengths and protects against potential threats to maintain and promote the health of the community. The present study aimed to design a crisis resilience model based on the institutionalization of organizational learning for managers and experts.

METHODS: The present exploratory analysis study was performed on a sample size of 215 which was calculated using Cochran's formula. In order to design a crisis resilience model based on the institutionalization of organizational learning, first, the general texts of applied sociology and scientific databases were studied. Afterward, 450 concepts regarding the general institutionalization of culture were extracted and provided for the experts in universities and the Red Crescent Society using the Delphi method. Subsequently, the experts agreed upon 73 concepts regarding the institutionalization of the culture of resilience based on organizational learning. Finally, a questionnaire was prepared based on these 73 concepts.

FINDINGS: The first step was the performance of the exploratory factor analysis in the SPSS version22 software on 73 concepts which resulted in the emergence of five main components, including management, objectives, culture, strategy, and education of crisis resilience. These components were identified and named according to the review of the related literature. In the second stage, the DEMATEL technique and software were used to determine the importance and effectiveness of the criteria. Eventually, in the third stage, the LISREL software was used to design the crisis resilience model based on the institutionalization of organizational learning.

CONCLUSION: First, the exploratory factor analysis was performed in SPSS software version22 and the effectiveness and susceptibility of each factor were determined using the DEMATEL software. Subsequently, the model components, including the culture, strategy, education, and objectives of resilience were used as the input of LISREL software and the resilience model was designed based on the institutionalization of organizational learning.
\end{abstract}

Original Article

Keywords: Crisis Situations; Institutionalization; Organizational Learning; Resilience.

How to cite this article: Ebrahimi A. Design of a Resilience Model Based on the Institutionalization of Organizational Learning in the Red Crescent Society. Sci J Rescue Relief 2020; 12(1): 35-45.

\section{Introduction}

$\mathrm{I}$ $\mathrm{n}$ the present study, crisis resilience based on the institutionalization of organizational learning is one of the studied components. Crisis resilience leads to the internalization of organizational strategies, assumptions, and norms in order to achieve productivity and meet the expectations and is stored in the organizational memory. Organizational resilience is a determining factor in the long-term performance and survival of the organization as a society (1).

Institutionalization of organizational learning is another component of the present study. Institutionalization of organizational learning leads to the internalization of organizational strategies, assumptions, and norms in order to achieve productivity and meet the expectations and is stored in the organizational memory. It also

1-PhD, Human Resource Management, Bafgh Azad University, Yazd, Iran

Correspondence to: Alireza Ebrahimi, Email: Ealireza88@yahoo.com 
provides the best method for the maintenance of success at length, provision of a response to threats, usage of opportunities, and innovation in various ways (2). Moreover, the institutionalization of organizational learning is an influential factor in organizational success and a source of resilience for the employee (3). It is a belief that provides the deepest and most enduring response to social influence and is based on the desire to display the right behavior and thoughts and its acceptance offers an inner reward (4).

The present study aimed to design a crisis resilience model based on the institutionalization of organizational learning. Therefore, the crisis resilience based on the institutionalization of organizational learning was discussed in order to be used in the Red Crescent Organization as one of the most important crisis management organizations in the country. Capability of the Red Crescent staff depends on their acceptance of the education and experience as well as their selfeducation by the observation of the resilience of the managers and supervisors in the organization during search and rescue operations and the specialized training provided by the Red Crescent Society.

Given the challenges facing the aid organizations, the cumulative complexity of natural disasters, the advancement of technology, and the need for managers to be resilient in times of crisis, it is important to note the extent of the improvement of employee resilience. Therefore, the present research aimed to determine if a crisis resilience model can be designed based on the institutionalization of organizational learning.

The term resilience refers to the increase in the tolerance of employees in times of crisis and disaster. Furthermore, institutionalization means the internalization of a value or a belief that is the most stable factor in social influence on individuals and is based on the desire to improve the behaviors and thoughts of the employee and the belief that it will result in an internal reward (9).

Stapinski et al. stated that the term institutionalization should not be confused with obedience and imitation. In obedience, one seeks rewards and tries to avoid punishment, and this behavior lasts as long as there is a promise of reward or the threat of punishment. Imitation refers to the attempt of trying to be like an influential person which, like obedience, is not based on inner satisfaction. The aim of imitation is to create a satisfying relationship with the person(s) one tries to imitate and one believes in what $\mathrm{s} / \mathrm{he}$ is trying to imitate; however, it is not permanent (12). It has been reported that employees with high resilience set higher personal goals and are more committed to those goals than those with low resilience (5).

Lazario (2015) examined the effect of tolerance on the motivation of employees and found that tolerance acted as a sample and selfmotivation since after observing the result of their tolerance, the employees set new goals that motivate them to make effort and show perseverance. In the end, high resilience creates a good feeling, while the lack of it causes an unpleasant feeling in the employees (11).

In a study, Gorbatov (2018) measured the ability to internalize organizational learning in managers of government, private, and multinational organizations and examined the relationship between the internalized organizational learning ability and organizational performance. The data of the above-mentioned study were randomly collected from 612 industrial managers in India. The results indicated a crisis regarding the ability to internalize organizational learning in Indian managers. Moreover, it was revealed that the managers of IT departments and multinational companies were the most capable of internalizing organizational learning, while managers of engineering departments were the least capable in this regard (6).

Rabindra et al. (2017) in a study investigated the mediating role of organizational learning ability on organizational resilience. They found that organizational learning ability has an important role in organizational resilience (7).

Myburgh (2014) performed a study titled "Development and Validity of the Scale of Resilience and Development of Organizational Learning of School Principals". In the aforementioned study, it was found that the required structure consisted of eight factors, including the creation of an appropriate organizational structure, guidance, and management of a resilient organization, self-assessment of the employees to improve their performance, conflict management, investigation of practices for classroom resilience, adherence to community and the required policies, and education of observation and resilient leadership (10).

Morris (2015) in a study found that men are on 
average more resilient than women. This gender difference peaks around the age of 30 and decreases in later years (12). Morris et al. (2015) in a study about the institutionalization of organizational citizenship culture identified the mediation of resilience, components of organizational commitment, organizational pride, organizational myths, and succession culture as the criteria of the model for the institutionalization of organizational culture (8).

Based on the results of the above-mentioned studies, it can be concluded that the organizations that institutionalized the orientation of resilience are more likely to experience better organizational growth, development, and performance which increases the resilience of their employees. The present study aimed to design a crisis resilience model based on the institutionalization of organizational learning of managers and experts for the first time. It is hoped that it can help managers to have better individual and organizational performance in society.

\section{Methods}

The present research aimed to design a crisis resilience model based on the institutionalization of organizational learning of managers and experts in the Red Crescent Society throughout the country. Regarding the method of the research, the researcher searched books, scientific texts, websites, and scientific and research journals for the component of resilience and institutionalization of organizational learning; however, the required data could not be found. Therefore, the studies in the field of social sciences and sociology were reviewed in terms of the institutionalization of the culture of resilience and the culture of institutionalization.

In total, 480 components were extracted from these studies which were provided for the experts in universities and the Red Crescent Organization through the Delphi method. Finally, about 73 concepts regarding the institutionalization of the culture of organizational learning and resilience were agreed upon by the experts, and a questionnaire was prepared accordingly. Subsequently, a crisis resilience model based on the institutionalization of organizational learning was designed in three stages by three software.

Step 1: Exploratory Factor Analysis

The SPSS software (which version?) was used to determine the index factors and effective concepts in the design of the model. Exploratory factor analysis is a method that is often used to detect and measure the hidden variance and covariance in the observed measurements. The researchers realized that the exploratory factor analysis can be quite useful in the early stages of the experiment or questionnaire development.

This type of analysis can be calculated in SPSS software version 22 . According to the exploratory factor analysis on 73 concepts, 5 components were identified as the main components according to the literature review. They included management components, objectives, culture, strategy, and resilience education. Each component consists of the variables that will be mentioned below.

\section{Stage 2: Application of DEMATEL Technique and Software}

In the face of complex issues, it is very important for the researcher to explore the interrelationships between the indicators. One of the basic issues that the researchers tackle is modeling the interrelationships between different indicators. One of the suitable methods for structuring is the use of DEMATEL software which is one of the cognitive methods of structuring decision-making problems.

DEMATEL method is based on the graph theory and we can divide the criteria into cause and effect groups to better understand the cause and effect interactions and draw a network of interactions. In this model, groups, criteria, and relationships were the graph edges. After forming the matrix, the calculations were performed on the matrix based on the following steps.

\section{A) Normalization of the matrix}

The following equation was used for normalization:

$$
\boldsymbol{N}=\boldsymbol{z A}
$$

Equation 1.

In this equation, $\mathrm{A}$ is a pairwise comparison matrix (PCM) and the value of $\mathrm{z}$ is calculated using equation 2 :

$$
z=1 / \max \left\{\max _{1 \leq S} \sum_{j=1}^{n} a_{\overline{\bar{p}}}, \max _{\mathbb{L} j \leq n} \sum_{i=1}^{n} a_{\bar{y}}\right\}
$$

Equation 2.

The $\mathrm{N}$ is the unscaled matrix of the pairwise 
comparison of the criteria.

\section{B) Matrix of interrelationships}

Matrix of interrelationships between the criteria was calculated using the following equation:

$$
\begin{aligned}
\boldsymbol{T} & =\boldsymbol{N}+\boldsymbol{N}^{2}+\ldots+\boldsymbol{N}^{k} \\
& =\boldsymbol{N}\left(\boldsymbol{I}+\boldsymbol{N}+\ldots+\boldsymbol{N}^{k-1}\right)\left[(\boldsymbol{I}-\boldsymbol{N})(\boldsymbol{I}-\boldsymbol{N})^{-1}\right] \\
& =\boldsymbol{N}\left(\boldsymbol{I}-\boldsymbol{N}^{k}\right)(\boldsymbol{I}-\boldsymbol{N})^{-1}=\boldsymbol{N}(\boldsymbol{I}-\boldsymbol{N})^{-1}
\end{aligned}
$$

Equation 3.

Which is summarized as:

$$
T=N(I-N)^{-1}
$$

Equation 4.

Subsequently, the DEMATEL technique was coded and written in MATLAB software version22in order to determine the importance and influence of the components that underlie the design of the model for the institutionalization of organizational learning.

In the third stage, LISREL software was used to design a crisis resilience model based on the institutionalization of organizational learning.

\section{Statistical population and sample of the research}

The statistical population consisted of managers and experts of the Red Crescent Society of the country, including 31 general managers and 456 managers from across the country. The sample size was estimated using the Cochran formula.

$$
n=\frac{\frac{z^{2} p q}{d^{2}}}{1+\frac{1}{N}\left(\frac{z^{2} p q}{d^{2}}-1\right)}
$$

Equation 5.

In this formula:

n: sample size

$\mathrm{N}$ : all the Red Crescent managers

$\mathrm{t}$ or $\mathrm{z}$ : percentage error of acceptable reliability coefficient

$\mathrm{p}$ : the ratio of the population with a certain trait (e.g., male population)

q (1-p): a ratio of the population without a definite attribute (e.g., female population)

$\mathrm{d}$ : desired precision of the estimate or the acceptable error in the estimate
Table 1. Reliability of the questionnaires based on the Cronbach's alpha

\begin{tabular}{|ccc|} 
Column & Questionnaire & $\begin{array}{c}\text { Cronbach's } \\
\text { alpha }\end{array}$ \\
\hline 1 & Resilience component & 0.82 \\
2 & $\begin{array}{c}\text { Institutionalization of } \\
\text { organizational learning }\end{array}$ & 0.73 \\
\hline
\end{tabular}

According to the above-mentioned formula, for a sample size with a population gap of 0.5 (i.e., half of the population has a certain trait, while the other half do not); the value of $\mathrm{z}$ should usually be 96.1 and $d$ can be 0.01 or 0.05 . To minimalize the error, we consider $\mathrm{p}=\mathrm{q}=0.5$. In the aforementioned formula, the sampling was performed with a confidence level of $25 \%$ and a $5 \%$ error while the value of $\mathrm{p}$ and $\mathrm{q}$ were considered to be $50 \%$ using the precautionary method. The error of the measuring instrument was $5 \%$; therefore, the sample size was calculated at 215 samples using the above formula.

\section{Data collection tools}

In the present study, the resilience questionnaires developed by Vergo et al. (2017) and Patton (2014) were used to collect the required data. This standard questionnaire which consists of 17 items shows how one organizes and implements the methods needed to achieve the expected situations.

In this research, to determine the reliability of each questionnaire separately, a preliminary study was performed on 30 subjects. Subsequently, the collected data were extracted and the Cronbach's alpha values were calculated which are summarized in the table 1 , which indicates the high accuracy and reliability of the questionnaires.

\section{Findings}

The main research question (i.e., is it possible to design a crisis resilience model based on the institutionalization of organizational learning) can be answered based on the findings.

In order to design a crisis resilience model based on the institutionalization of organizational learning of managers and experts, first, the Delphi method was used to find out the opinion of experts regarding the institutionalizing of the components of organizational learning and culture of resilience. Finally, the experts agreed upon 87 out of the 480 concepts. First, the stages of 
designing a crisis resilience model based on the institutionalization of organizational learning are described below.

\section{Step 1. Exploratory factor analysis}

Based on the results of this step, there were five outputs. The first output revealed the value of the software index of the Kaiser-Meyer-Olkin (KMO) measure of sampling adequacy, the value of the Bartlett test statistic (which is an approximation of the Chi-square test), the degree of freedom, and the p-value. Since the value of the software index of KMO was 0.746 (between 0.51), the number of subjects (sample) was sufficient for factor analysis. Moreover, the p-value of the Bartlett test in the software was less than 5\% which indicated that factor analysis was appropriate for the identification of the factor structure and model. Furthermore, the assumption that the correlation matrix was known was rejected.

The second output showed the initial and extracted communalities. Since the initial communalities are the communalities before extracting the concepts, all of them equal one. The larger the values of the extracted communalities, the better the extracted concepts represent the variables. However, if the values of the extracted communalities for the variables were small, it can be omitted. Nevertheless, it should be noted that the variables should be omitted step by step and that the variable which has the least amount of extraction should be deleted first.

The third output, which was called the rotation sums of squared loadings in the software, revealed the eigenvalues of extraction factors by rotation. In this analysis, five factors had an eigenvalue greater than three and remained in the analysis. (Since there were too many variables the number three was selected to limit the number of factors which certainly increased the number of effective variables in each factor. However, if the number one had been selected, the number of factors would have increased significantly which would have reduced the influential variables in each factor. Reduction of the influential variables in each factor would make it difficult to name these factors and increase the possible effect of one variable on several factors resulting in the incorrect analysis of the impact of a variable on a factor. Therefore, the number of factors is limited which makes it possible to perform the correct analysis).

These five factors can explain approximately $50 \%$ of the variance variability of the variables. It should be noted that in the rotation of the remaining factors, the ratio of the total change explained by these five factors was almost constant. However, unlike the non-rotation method, anyway, in the factor rotation method, each factor is a fifth higher percentage of change. (Approximately 50) Explains that the factors explain almost the same proportion of changes. This feature is called the Varimax rotation which distributes the changes evenly among the factors.

The third output contained three parts. The first part, which was called the initial eigenvalues, determined the factors that remained in the analysis (factors that had an eigenvalue of less than one were excluded from the analysis). Factors that were excluded from the analysis were the factors whose presence did not further explain the variance. The second part, entitled extraction sums of squared loadings, was about the eigenvalues of extraction factors without rotation.

The fourth output showed the component matrix, which included the factor loadings of each variable. Moreover, the fifth output showed the rotated matrix of the concepts. The higher absolute values of the coefficients indicate the greater role of the relevant factor in the total variations of the variable.

As mentioned in the third part of factor analysis, in this analysis, five factors have eigenvalues greater than three and remain in the analysis. (Since there were too many variables the

Table 2. Varimax rotation of the concepts of the model of institutionalization of organizational learning

\begin{tabular}{|cccccccccc|}
\hline & \multicolumn{3}{c}{ Initial eigenvalues } & \multicolumn{3}{c}{ Least squares } & \multicolumn{2}{c}{ Rotation sums of squared loadings } \\
Component & Total & $\begin{array}{c}\text { Percentage } \\
\text { of variance }\end{array}$ & $\begin{array}{c}\text { Cumulative } \\
\text { percentages }\end{array}$ & Total & $\begin{array}{l}\text { Percentage } \\
\text { of variance }\end{array}$ & $\begin{array}{c}\text { Cumulative } \\
\text { percentages }\end{array}$ & Total & $\begin{array}{c}\text { Percentage } \\
\text { of variance }\end{array}$ & $\begin{array}{c}\text { Cumulative } \\
\text { percentages }\end{array}$ \\
\hline 1 & 18.930 & 25.931 & 25.931 & 18.930 & 25.931 & 25.931 & 16.040 & 21.972 & 21.972 \\
2 & 6.725 & 9.212 & 35.143 & 6.725 & 9.212 & 35.143 & 8.197 & 11.229 & 33.201 \\
3 & 4.458 & 6.107 & 41.251 & 4.458 & 6.107 & 41.251 & 4.700 & 6.438 & 39.639 \\
4 & 3.626 & 4.967 & 46.217 & 3.626 & 4.967 & 46.217 & 4.534 & 6.210 & 45.89 \\
5 & 3.423 & 4.688 & 50.906 & 3.423 & 4.688 & 50.906 & 3.691 & 5.056 & 50.906 \\
\hline
\end{tabular}


number three was selected to limit the number of factors which certainly increased the number of effective variables in each factor. However, if the number one had been selected, the number of factors would have increased significantly which would have reduced the influential variables in each factor. Reduction of the influential variables in each factor would make it difficult to name these factors and increase the possible effect of one variable on several factors resulting in the incorrect analysis of the impact of a variable on a factor. Therefore, the number of factors is limited which makes it possible to perform the correct analysis).

These five factors can explain approximately $50 \%$ of the variance variability of the variables. It should be noted that in the rotation of the remaining factors, the ratio of the total change explained by these five factors was almost constant. However, unlike the non-rotation method, although, in the factor rotation method, each factor is a fifth higher percentage of change. (Approximately 50) Explains that the factors explain almost the same proportion of changes.

Given the factor analysis of 73 effective concepts in the institutionalization of the culture of organizational learning, five components were the main components of identification of the remaining factors. It is difficult to interpret the factor loadings without rotation; therefore, we rotated the factors to increase their interpretability.

These five factors should be named according to the literature review. Each component included a concept that will be explained. Component number one (resilience culture) included the concepts of creation of a culture of learning together, creation of a culture of trial and error and ways to improve them, creation of the culture of trust in the organization, creation of creative dialogue in the organization, usage of the method of coaching in the organization to enhance learning, creation of a culture of asking questions in the organization, and creation of a unit for the scientific and practical problems of work.

Component number two (resilience management) included (managerial) concepts of creation of a clear vision to promote learning, administration of tests to evaluate learning, make the learning of the staff influential on their career, creation of problem-solving skills and open attitudes, managers' commitment to the improvement of education, identification of the source of information flows to take advantage of it, reduction of the number of middle managers for the quick access to information.

Component number three (resilience strategy) included modification of cumbersome administrative regulations regarding education, the definition of organizational learning as a job requirement, development of learning strategies in the organization, improvement of individual abilities regarding the use of modern technologies, consideration of organizational learning as intangible assets of the organization, inclusion of an education plan in the articles of association and the strategic plan.

Component number four (resilience education) included encouragement of team learning, creation of a group on social media for access to educational resources, the involvement of people to assist learning, consideration of the people's perception of learning, cooperation with knowledge enterprise, and knowledge acquisition and learning facilitation system.

Component number five (resilience objectives) included the concepts of participation of individuals in the establishment of objectives, defiance of the old norms in the organization, creation of a common understanding of organizational learning, emphasis on goals, encouragement of superior products and services, belief in individual abilities, and documentation and expression of the relationship between new learning behaviors and organizational successes.

\section{Step 2. Application of DEMATEL technique and software}

The DEMATEL technique and software were used for the determination of the level of importance and effectiveness of the criteria for designing a crisis resilience based on the institutionalization of organizational learning.

\section{Selection of components of the crisis resilience model based on institutionalization of organi- zational learning}

First, of the importance and effectiveness of the criteria (components) were determined through the DEMATEL technique and software. Subsequently, in order to evaluate the effect of the criteria on the goal, which was to design a model for the institutionalization of organizational learning, five criteria were used that are 
Table 3. The Criteria

\begin{tabular}{|ccc|} 
Name & Symbol & Number \\
Resilience culture & $C_{1}$ & 1 \\
Resilience management & $C_{2}$ & 2 \\
Resilience strategy & $C_{3}$ & 3 \\
Resilience education & $C_{4}$ & 4 \\
Resilience objectives & $C_{5}$ & 5 \\
\hline
\end{tabular}

introduced in Table 3.

The criteria were evaluated by 25 experts. Table 3 shows the pairwise comparison of the experts. In these matrices, $\mathrm{x}_{\mathrm{ij}}$ is the opinion of each expert and $\mathrm{x}_{\mathrm{ii}}=(\mathrm{i}=1,2,3, \ldots, \mathrm{n})$ is equal to zero (the main diameter is zero). In order to consider the opinion of all the experts, their arithmetic mean was calculated according to equation 6.

$Z=\frac{x^{1}+x^{2}+x^{3}+\ldots+x^{p}}{p}$

Equation 6.

In this formula, $\mathrm{p}$ is the number of experts and $\mathrm{x}^{1}, \mathrm{x}^{2}, \mathrm{x}^{\mathrm{p}}$ are the PCM of expert ${ }^{1}$, expert $^{2}$ and expert $^{\mathrm{p}}$, respectively.

Table 4 summarizes the mean values of pairwise comparisons.

Equations 7 and 8 were used to normalize the resulting matrix:

$H_{i j}=\frac{z_{i j}}{r}$

Equation 7.
The $\mathrm{r}$ is calculated using the following formula:

$r=\max _{1 \leq i \leq n}\left(\sum_{j=1}^{n} z_{i j}\right)$

Equation 8.

After calculation of the above matrixes, the total-relation fuzzy matrix was obtained according to the equation 9 .

$T=\lim _{k \rightarrow+\infty}\left(H^{1}+H^{2}+\cdots+H^{k}\right)=H \times(I-H)^{-1}$

Equation 9.

In this formula, I represents the identity matrix. The table 6 shows the T matrix.

The next step was to obtain the sum of the rows and columns of the T matrix. Sum of the rows and columns were calculated according to equation 10 and 11.

$$
\begin{array}{ll}
\text { (D ) })_{n \times 1}=\left[\sum_{j=1}^{n} T_{i j}\right]_{n \times 1} & \text { Equation } 10 . \\
\text { (R ) })_{1 \times n}=\left[\sum_{i=1}^{n} T_{i j}\right]_{1 \times n} & \text { Equation } 11 .
\end{array}
$$

Here, $D$ and $R$ are the matrixes $n \times 1$ and $1 \times n$, respectively.

Table 4. Mean values of the opinions of all the experts

\begin{tabular}{|c|c|c|c|c|c|}
\hline Mean values of the opinions of the experts & $\mathbf{C}_{\mathbf{1}}$ & $\mathbf{C}_{\mathbf{2}}$ & $\mathbf{C}_{\mathbf{3}}$ & $\mathbf{C}_{\mathbf{4}}$ & $\mathbf{C}_{\mathbf{5}}$ \\
\hline $\mathrm{C}_{1}$ & 0 & 2.84 & 2.81 & 2.8 & 2.60 \\
$\mathrm{C}_{2}$ & 1.28 & 0 & 2.48 & 2.6 & 2.64 \\
$\mathrm{C}_{3}$ & 1.36 & 1.24 & 0 & 2.13 & 2.56 \\
$\mathrm{C}_{4}$ & 1.27 & 1.26 & 1.27 & 0 & 2.68 \\
$\mathrm{C}_{5}$ & 1.23 & 1.27 & 1.23 & 1.26 & 0 \\
\hline
\end{tabular}

Table 5. Normalized submatrices

\begin{tabular}{|c||cccc|c|}
\hline Normalized submatrices & $\mathbf{C}_{\mathbf{1}}$ & $\mathbf{C}_{\mathbf{2}}$ & $\mathbf{C}_{\mathbf{3}}$ & $\mathbf{C}_{\mathbf{4}}$ & $\mathbf{C}_{\mathbf{5}}$ \\
\hline $\mathrm{C}_{1}$ & 0 & 0.26 & 0.25 & 0.25 & 0.24 \\
$\mathrm{C}_{2}$ & 0.12 & 0 & 0.22 & 0.24 & 0.24 \\
$\mathrm{C}_{3}$ & 0.12 & 0.11 & 0 & 0.19 & 0.23 \\
$\mathrm{C}_{4}$ & 0.12 & 0.11 & 0.11 & 0 & 0.24 \\
$\mathrm{C}_{5}$ & 0.11 & 0.11 & 0.11 & 0.11 & 0 \\
\hline
\end{tabular}

Table 6. Total relation matrix

\begin{tabular}{|c|cccc|c|}
\hline Total relation matrix & $\mathbf{C}_{\mathbf{1}}$ & $\mathbf{C}_{\mathbf{2}}$ & $\mathbf{C}_{\mathbf{3}}$ & $\mathbf{C}_{\mathbf{4}}$ & $\mathbf{C}_{\mathbf{5}}$ \\
\hline $\mathrm{C}_{1}$ & 0.30 & 0.56 & 0.62 & 0.67 & 0.75 \\
$\mathrm{C}_{2}$ & 0.35 & 0.29 & 0.51 & 0.56 & 0.64 \\
$\mathrm{C}_{3}$ & 0.31 & 0.34 & 0.27 & 0.47 & 0.56 \\
$\mathrm{C}_{4}$ & 0.29 & 0.32 & 0.35 & 0.28 & 0.53 \\
$\mathrm{C}_{5}$ & 0.25 & 0.28 & 0.31 & 0.34 & 0.28 \\
\hline
\end{tabular}


Table 7. Importance and effectiveness of the criteria

\begin{tabular}{|c|c|c|}
\hline Criteria & Di+Ri & Di-Ri \\
\hline Criteria 1 & 4.40 & 1.39 \\
Criteria 2 & 4.15 & 0.56 \\
Criteria 3 & 4.02 & -0.11 \\
Criteria 4 & 4.08 & -0.54 \\
Criteria 5 & 4.23 & -1.30 \\
\hline
\end{tabular}

The next step was to determine the importance of the indicators $\left(D_{i}+R_{i}\right)$ and the relationship between the criteria $\left(D_{i}-R_{i}\right)$. If $D_{i}-R_{i}>0$, the relevant criterion is effective and if $D_{i}-R_{i}<0$, the relevant criterion is susceptive. The table 7 shows Di-Ri and Di+Ri.

Step 3. Design of a crisis resilience model based on the institutionalization of organizational learning using LISREL software

There are five underlying components for the design of the model, including components of resilience strategy, resilience objectives, resilience management, resilience education, and resilience culture. Moreover, the importance and effectiveness of the criteria were determined through the DEMATEL technique and software. The output of DEMATEL software was used as input for LISREL software for the design of a crisis resilience model based on the institutionalization of organizational learning. (Figure 1)

In this model, the values of the goodness of fit index and the adjusted goodness of fit index were 0.92 and 0.86 , respectively. Closeness of the values of the indicators indicates the better fit of the model; therefore, the proposed model had a good fit. The following table summarizes the fit indicators of the crisis resilience model based on the institutionalization of organizational learning.

\section{Factor analysis of resilience questionnaire based on the institutionalization of organizational learning}

The standard factor loading of confirmatory factor analysis for the evaluation of the strength of the relationship between each factor (latent variable) and its observed variables (i.e., questionnaire items, including the resilience culture, resilience education, resilience strategy, and resilience objectives) in all cases was greater than 0.3 . Therefore, the operating structure of the questionnaire can be confirmed.

After calculating the standard factor loading, a significance test should be performed. Based on the results shown in the figure below, the factor loading of the t-statistic of the evaluation indices of each of the studied aspects with a 5\% confidence level was greater than 1.96.

The normal chi-square index for the abovementioned model was calculated as follows:

$$
\frac{x^{2}}{d f}=\frac{247.13}{113}=2.187
$$

Moreover, since the root mean square approximation index was 0.038 , the model has a good fit. Moreover, the other goodness-of-fit indices were within the accepted range which is shown in Table 9.

RMSEA: root mean square error of approximation, GFI: goodness of fit index, AGFI: adjusted goodness of fit index, NFI: normed fit index, NNFI: non-normed fit index, IFI: incremental fit index

\section{Discussion and Conclusion}

The present study aimed to design a resilience model based on the institutionalization of organizational learning in the Red Crescent Society. The main research question was whether it is possible to design a resilience model based on the institutionalization of organizational learning? The answer to this question can be summarized as follows: first, after the collective agreement on 73 concepts through the Delphi method, five main components were identified, including resilience management, resilience objectives, resilience culture, resilience strategy, and resilience education. The components were named according to the review of the related literature.

Afterward, the five components of the crisis resilience model design as well as the level of importance and effectiveness of the criteria, which were determined through the DEMATEL technique and software, were used as an input or the LISREL software to design the research model.

Results of a study performed by DeWitz SJ, Woolsey ML et al., define resilience capacity as a capacity that continuously overcomes challenges and identifies new opportunities (12) which is similar to the component of resilience strategy in the present study. Moreover, based on the findings of a study conducted by Stapinski LA, the capacity of a system is defined by its potential to 


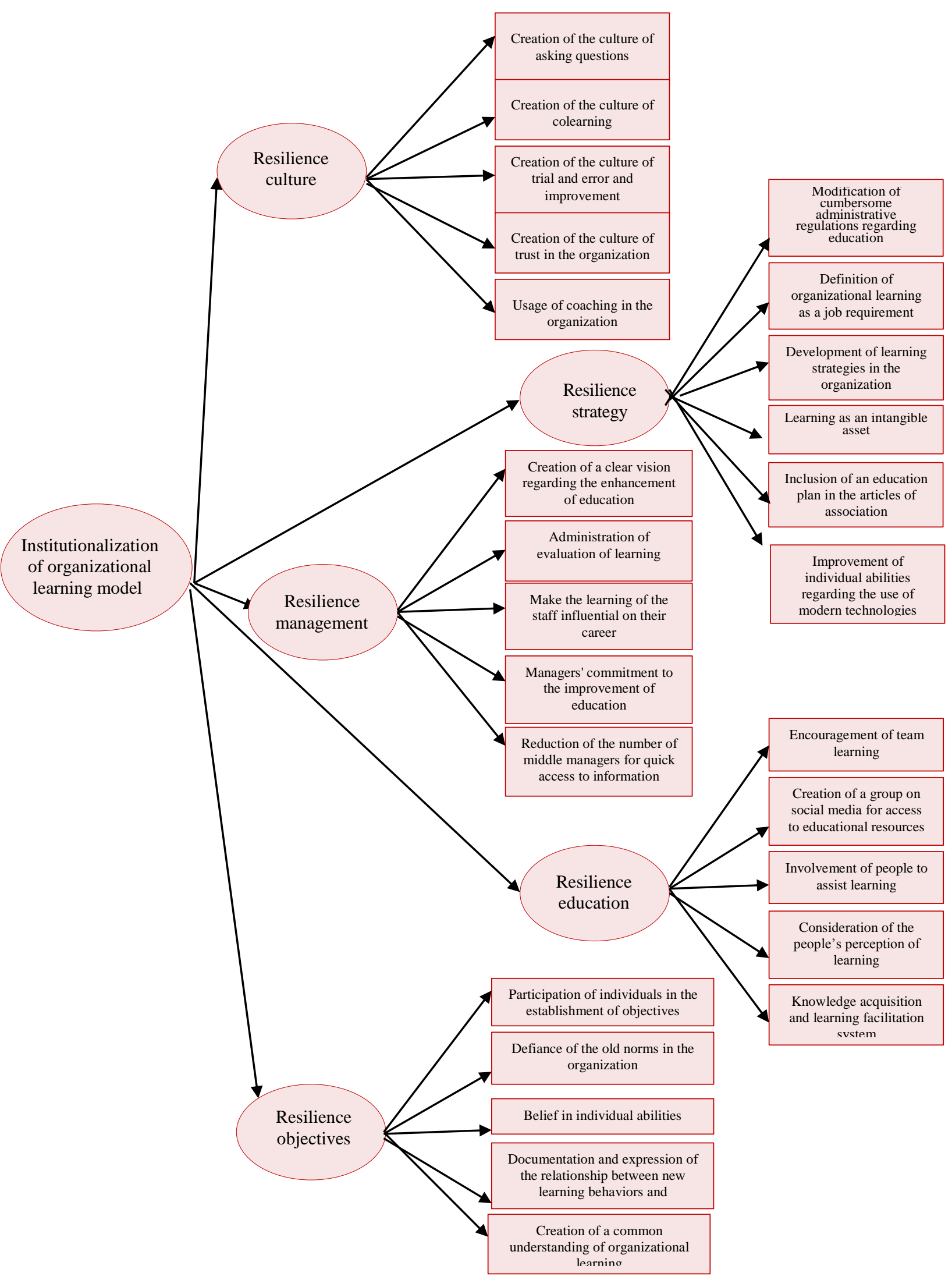

Figure 1. Research model and the relationship between research variables in the structural equation model 
Table 8. Fit indices of the crisis resilience model based on the institutionalization of organizational learning

\begin{tabular}{|cc|} 
Fit indices & Estimation \\
Goodness of fit index & 0.92 \\
Adjusted goodness fit index & 0.86 \\
p-value & 1.000 \\
Root mean square error of approximation & 0.083 \\
Chi-square $\left(\chi^{2}\right)$ & 185.8 \\
Degree of freedom & 85 \\
\hline
\end{tabular}

Table 9. Goodness of fit indices of factor analysis of resilience questionnaire $\mathrm{P}<0.05$

\begin{tabular}{|ccccc|cc|}
\hline Goodness of fit & RMSEA & GFI & AGFI & NFI & NNFI & IFI \\
\hline Acceptable values & $<0.1$ & $>0.9$ & $>0.9$ & $>0.9$ & $>0.9$ & $0-1$ \\
Calculated values & 0.038 & 0.96 & 0.94 & 0.98 & 0.91 & 0.95 \\
\hline
\end{tabular}

adapt through stability or change to achieve an acceptable level of functionality and structure, defiance of the foundation of authoritarian values in the organization, comprehensive perception of organizational learning, focus on goals, and pride in achievements (5) which is consistent with the component of resilience objectives in the present study. One model of individual resilience is the Antonovsky's model which consists of three components:

\section{Comprehensibility}

The extent to which a person understands the world in an orderly manner and understands the problems s/he faces clearly.

\section{Manageability}

The degree to which a person believes that the resources needed to succeed are at their disposal or at the disposal of others on whom the person relies.

\section{Significance}

The extent to which a person feels that the problems and needs posed by life are valuable challenges of commitment and engagement (5) that conform to the five components of the present research model, including learning objectives, learning management, learning strategy, and learning culture.

According to the results, it is suggested that the Red Crescent Society develop strategies and perspectives based on the five underlying components of crisis resilience in order to improve crisis resilience in its employees.

\section{Acknowledgments}

The author would like to express his gratitude to the officials and reviewers of the Journal of Rescue and Relief for their responsible answers and great patience.

\section{Conflict of Interests}

The authors declare that they have no conflict of interest regarding the publication of the current study.

\section{References}

1. Aguinis $\mathrm{H}$, Kraiger $\mathrm{K}$. Benefits of training and development for individuals and teams, organizations, and society. Ann Rev Psychol 2009; 60: 451-74.

2. Nafukho FM, Graham CM, Muyia MH. Determining the relationship among organizational learning dimensions of a small-size business enterprise. J Eur Indust Train 2009; 33(1): 32-51.

3. Lin HF. Effects of extrinsic and intrinsic motivation on employee knowledge sharing intentions. J Inform Sci 2007; 33(2): 135-49.

4. Redmond BR. Self-efficacy and social cognitive theories. Confluence. Available at: URL: https://wikispaces.psu.edu/display/psych484/7.+self efficacy+and+social+cognitive+theories; 2016.

5. Stapinski LA, Abbott MJ, Rapee RM. Evaluating the cognitive avoidance model of generalized anxiety disorder: Impact of worry on threat appraisal, perceived control, and anxious arousal. Behav Res Ther 2010; 48(10): 1032-40.

6. Gorbatov S, Khapova SN, Lysova EI. Personal branding: interdisciplinary systematic review and research agenda. Front Psychol 2018; 9: 2238.

7. Pradhan RK, Jena LK, Singh SK. Examining 
the role of emotional intelligence between organizational learning and adaptive performance in Indian manufacturing industries. J Workplace Learn 2017; 29(3): 235-47.

8. Morris MW, Hong YY, Chiu CY, Liu Z. Normology: integrating insights about social norms to understand cultural dynamics. Organ Behav Hum Dec Proc 2015; 129: 1-3.

9. Myburgh W, Watson MB, Foxcroft CD. Development and validation of a managerial decision making self-efficacy questionnaire. SA J Indust Psychol 2015; 41(1): 1-15.

10. Riggs ML, Warka J, Babasa B, Betancourt R, Hooker S. Development and validation of selfefficacy and outcome expectancy scales for jobrelated applications. Educ Psychol Meas 1994; 54(3): 793-802.

11. Lăzăroiu G. Employee motivation and job performance. Linguistic Philosophical Investigation 2015; 14: 97-102.

12. DeWitz SJ, Woolsey ML, Walsh WB. College student retention: an exploration of the relationship between self-efficacy beliefs and purpose in life among college students. J Coll Stud Dev 2009; 50(1): 19-34.

13. Kimhi S, Eshel Y. Individual and public resilience and coping with long-term outcomes of war 1 . J Appl Biobehav Res 2009; 14(2): 70-89. 